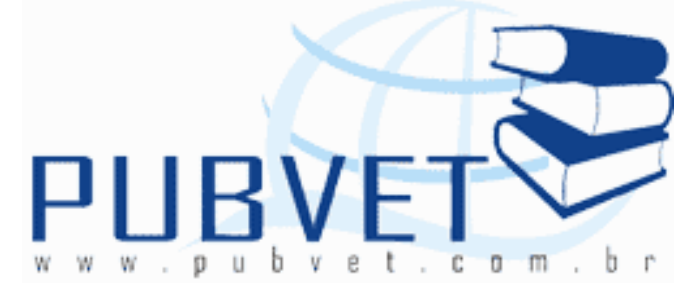

PUBVET, Publicações em Medicina Veterinária e Zootecnia.

\title{
Esqueletopia do cone medular do javali Sus scrofa scrofa (Linnaeus, 1758)
}

André Luiz Quagliatto Santos, Lucélia Gonçalves Vieira, Líria Queiroz Luz Hirano, Árthur Paulino Sanzo Kaminishi, Juliana dos Santos Mendonça, Thaís Carneiro Santos Rodrigues, Sthéfani Evangelista Siqueira.

Laboratório de Ensino e Pesquisa em Animais Silvestres (LAPAS), Universidade Federal de Uberlândia (UFU). Avenida Amazonas 2245, Jardim Umuarama, 38.405-302, Uberlândia-MG.

E-mail: quagliatto@famev.ufu.br

\section{Resumo}

A anestesia caudal foi proposta pela primeira vez em 1926 e diversas técnicas foram desenvolvidas para anestesiar os nervos espinhais lombares e sacrais. Este estudo determinou a esqueletopia do cone medular de Sus scrofa scrofa, utilizando 18 javalis de ambos os sexos, sendo 7 fêmeas e 11 machos, provenientes de um criatório no município de Romaria, Minas Gerais. Estes foram fixados em solução aquosa de formol à $10 \%$ e conservados em tanque de igual solução. Foram retirados os músculos da região dorsal à coluna vertebral, os arcos vertebrais e o tecido adiposo, expondo a medula espinhal e o cone medular. Houve variância na disposição deste entre os espécimes, sendo que os cones medulares dos javalis estenderam-se da vértebra $L_{4}$ até a terceira vértebra sacral $\left(S_{3}\right)$, com comprimento médio de $34,16 \mathrm{~mm} \pm 7,23$ 
mm. Conclui-se assim que o melhor local para anestesia epidural do membro pélvico em Sus scrofa scrofa é entre as vértebras $L_{4}$ e $S_{3}$.

Palavras-chave: anatomia, anestesia, cone medular, javalis.

\title{
Esqueletopia of conus boar of the Sus scrofa scrofa (Linnaeus, 1758)
}

\begin{abstract}
The caudal anesthesia was first proposed in 1926 and several techniques have been developed to anesthetize the lumbar and sacral spinal nerves. This study determined the medullary esqueletopia cone of Sus scrofa scrofa, using 18 boars of both sexes, 7 females and 11 males, from a farm in the municipality of Pilgrimage, Minas Gerais. These were fixed in aqueous formaldehyde $10 \%$ and stored in the same tank solution. Muscles were removed from the dorsal to the spinal column and the vertebral arches and adipose tissue, exposing the spinal cord and conus. There was variance in this provision among the specimens, and the medullary cones of boars extended to the $L_{4}$ vertebra to the fourth sacral vertebra $\left(S_{3}\right)$, with a mean length of $34.16 \mathrm{~mm} \pm 7.23 \mathrm{~mm}$. It follows that the best location for epidural anesthesia in the hindlimb Sus scrofa scrofa is between vertebrae $L_{4}$ and $S_{3}$.
\end{abstract}

Keywords: anatomy, anesthesia, medullary cone, boars.

\section{INTRODUÇÃO}

O javali (Sus scrofa scrofa) é considerado um ancestral do porco doméstico, sendo nativo da Ásia, África e Europa e introduzido pelo homem na América há muito tempo. Sua domesticação teve início há aproximadamente 5.000 anos (HRUBY; DEWY, 2004). Na América do Sul, estes animais foram soltos no Uruguai e Argentina, onde houve o cruzamento descontrolado com suínos domésticos (Sus scrofa domestica) (HRUBY; DEWY, 2004; BERGONSO, 2010). 
Anatomicamente o javali se assemelha ao suíno doméstico e vive em grupos tendo em média 20 indivíduos, entre machos jovens e fêmeas com filhotes. Os machos adultos se unem ao grupo apenas na estação reprodutiva, disputando até a morte as fêmeas, sendo que 0 vencedor torna-se responsáveis pela proteção de até 8 javalinas (BERGONSO, 2010).

$O$ aumento no consumo da carne de javali têm ocorrido sobretudo devido a sua crescente importância econômica. Esta é classificada como alimento nobre, de sabor exótico, com menor quantidade de gordura e de excelentes preços de mercado (BERGONSO, 2010). No Brasil, nos últimos anos, houve um crescimento na criação de javalis, o que acarretou consequentemente a existência de várias doenças infecciosas (ECCO et al., 2009).

Um fator importante é a carência de trabalhos científicos na Medicina Veterinária sobre esta espécie animal (BERGONSO, 2010). Algumas pesquisas relacionadas à sua morfologia estão sendo desenvolvidas, no entanto, até o momento pouco se sabe sobre a anatomia de seu sistema neural e a descrição morfológica da porção terminal da medula espinhal. Esta apresenta relevante importância para o médico veterinário e para o biólogo, permitindo comparação a outras espécies domésticas e silvestres (ALBUQUERQUE et al., 2003a; ALBUQUERQUE et al., 2003b).

O sistema neural têm despertado um interesse especial nos pesquisadores desde muito tempo, e o cone medular do gênero Sus, quando descrita por autores, geralmente é considerada como a do suíno doméstico, devido à escassez de informações sobre a anatomia do javali. Apesar dos trabalhos desenvolvidos sobre os diferentes aspectos anatômicos atinentes ao sistema neural, ainda há muito por se acrescentar, principalmente contribuições que permitam desenvolver novas técnicas à aplicação de anestesia epidural. Essa é uma técnica anestésica consagrada, que tem como principais vantagens a segurança, eficiência e baixo custo (HALL, 1992). A ausência quase total de depressão cárdio-respiratória favorece sua indicação como técnica anestésica alternativa para animais de alto risco, onde a 
anestesia geral estaria contra-indicada (BEDNARSKI 2001). No entanto, paralelamente, a anestesiologia veterinária evoluiu de forma considerável nas últimas décadas, desde a introdução da anestesia caudal em 1926 por Benesch (1926), para anestesiar os nervos espinhais lombares e sacrais em bovinos (ST. CLAIR; HARDENBROOK, 1956).

Com o intuito de fornecer subsídios para o desenvolvimento de novas técnicas anestesiológicas, e ainda acrescentar informações mais específicas à literatura, este trabalho tem como objetivo determinar a extensão do cone medular, bem como suas relações com as vértebras da coluna vertebral, em Sus scrofa scrofa.

\section{MATERIAL E MÉTODOS}

Foram utilizados 18 javalis, de ambos os sexos, sendo 7 fêmeas e 11 machos, provenientes de um criatório no município de Romaria, Minas Gerais. Os animais foram fixados em solução de formaldeído a $10 \%$ e conservados em tanque de igual solução. Posteriormente, foram retirados os músculos da região dorsal à coluna vertebral, em seguida removeram-se os arcos vertebrais desta mesma região, como também o tecido adiposo epidural, juntamente com meninges, expondo-se a medula espinhal e o cone medular. Com a visualização do cone medular, utilizou-se um paquímetro (Starrett/125B) para efetuar as medições do comprimento total do cone medular, seu início e término em relação às vértebras. Os dados foram registrados em forma de tabelas, desenhos e fotografias.

\section{RESULTADOS E DISCUSSÀO}

Observou-se que o início do cone medular ocorreu com maior frequência ao nível da quinta vértebra lombar, sendo este evento encontrado 5 vezes nas espécimes analisadas $(27,77 \%)$. Este resultado difere do que foi observado em equinos e ovinos, com inicio do cone medular entre as vértebras $L_{5} / L_{6}$ (HALL, 
1992; LIMA; SANTOS, 1998). Comparando com espécies de preguiças, evidencia-se que na espécie Choloepus hoffmanni (preguiça-de-hoffmann) o cone medular teve início ao nível da articulação $L_{3} / L_{4}$ enquanto que em Bradypus variegatus (preguiça-comum), este ocorreu entre $\mathrm{L}_{4} / \mathrm{S}_{1}$ (AMORIM JÚNIOR et al., 2003). Em cães, o cone medular (limite caudal da medula espinhal) ocorre entre as vértebras $L_{6} / L_{7}$, sendo 0 espaço lombossacro recomendando como sítio para a anestesia epidural, semelhante aos gatos (HANDERSON, 1977; BHON, 1981).

$O$ término na maioria dos casos aconteceu na vértebra $S_{3}$, sendo esta encontrada em 9 dos 18 totais javalis analisados (50\%) (Figura 1). Contrariamente em relação a equinos e bovinos, nos quais ocorreram na $\mathrm{S}_{2}$ (BRUNI; ZIMMERL, 1947), nos coelhos também foi concluído o término do cone medular na vértebra $\mathrm{S}_{2}$ (LIMA; SANTOS, 1998).

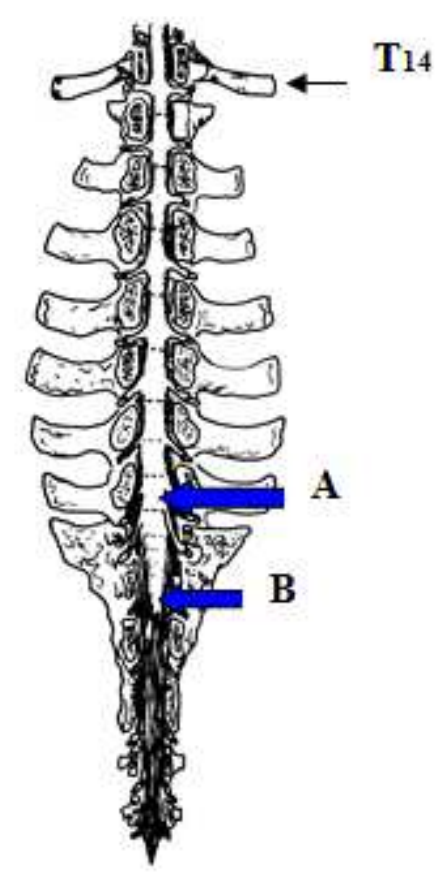

Figura 1: Vista dorsal do cone medular no canal vertebral, nas regiões lombar e sacral, demonstrando o inicio (A), em correspondência com L6, e o termino (B), na S3 de Sus scrofa scrofa. 
Em relação ao comprimento do cone medular, encontrou-se um valor de comprimento médio de 34,16 mm $\pm 7,23 \mathrm{~mm}$ (Tabela 1). Ao comparar com estudos já realizados, verifica-se que em ovinos sem raça definida esse comprimento é de $8,22 \mathrm{~cm}(82,2 \mathrm{~mm})$ (DYCE et al., 1997). Já em cavalos esse comprimento é de $10 \mathrm{~cm}$ (100mm) (RAO, 1990); enquanto que em coelhos a medida encontra é de $51 \mathrm{~cm}(51 \mathrm{~mm})$ (LIMA; SANTOS, 1998).

Através dos resultados obtidos pode-se observar que o cone medular apresenta terminações que variam em relação a espécie e a idade do animal, ocorrendo entre as últimas vértebras lombares e as primeiras sacrais (HOPKINS, 1935; HABEL, 1951; SEIFERLE, 1951; SHARMA et al., 1971; BRUNI; ZIMMERL, 1977; GETTY, 1986; SANTIAGO et al., 1990; RAO, 1990; RAO et al., 1993; SANTOS et al., 2001; DYCE et al., 2004; SANTOS et al., 2011).

Tabela 1: Comprimento do cone medular (C.C.M.) e esqueletopia do início e do término do cone medular de Sus scrofa scrofa. Uberlândia, 2005.

\begin{tabular}{cccc}
\hline $\begin{array}{c}\text { Número de } \\
\text { Observações }\end{array}$ & C.C.M. & $\begin{array}{c}\text { Início do } \\
\text { cone } \\
\text { medular }\end{array}$ & $\begin{array}{c}\text { Término } \\
\text { do cone } \\
\text { medular }\end{array}$ \\
\hline 1 & 29,80 & $\mathrm{~L}_{5}$ & $\mathrm{~S}_{2}$ \\
2 & 45,15 & $\mathrm{~L}_{5}$ & $\mathrm{~S}_{3}$ \\
3 & 25,40 & $\mathrm{~L}_{6}$ & $\mathrm{~S}_{2}$ \\
4 & 32,30 & $\mathrm{~L}_{6} \mathrm{~S}_{1}$ & $\mathrm{~S}_{3}$ \\
5 & 32,85 & $\mathrm{~L}_{6}$ & $\mathrm{~S}_{3}$ \\
6 & 33,70 & $\mathrm{~L}_{5}$ & $\mathrm{~S}_{3}$ \\
7 & 22,80 & $\mathrm{~L}_{5}$ & $\mathrm{~S}_{3}$ \\
8 & 41,90 & $\mathrm{~L}_{6}$ & $\mathrm{~S}_{3}$ \\
9 & 30,25 & $\mathrm{~L}_{4} \mathrm{~L}_{5}$ & $\mathrm{~S}_{1}$ \\
10 & 32,00 & $\mathrm{~L}_{6} \mathrm{~S}_{1}$ & $\mathrm{~S}_{3}$ \\
11 & 21,70 & $\mathrm{~L}_{6} \mathrm{~S}_{1}$ & $\mathrm{~S}_{3}$ \\
12 & 39,65 & $\mathrm{~L}_{5} \mathrm{~L}_{6}$ & $\mathrm{~S}_{2}$ \\
13 & 36,30 & $\mathrm{~L}_{5}$ & $\mathrm{~S}_{1} \mathrm{~S}_{2}$ \\
14 & 34,60 & $\mathrm{~L}_{5} \mathrm{~L}_{6}$ & $\mathrm{~S}_{1} \mathrm{~S}_{2}$ \\
15 & 46,00 & $\mathrm{~L}_{5} \mathrm{~L}_{6}$ & $\mathrm{~S}_{2}$ \\
16 & 33,60 & $\mathrm{~L}_{4}$ & $\mathrm{~S}_{1}$ \\
17 & 45,25 & $\mathrm{~L}_{6}$ & $\mathrm{~S}_{3}$ \\
18 & 31,65 & $\mathrm{~S}_{1}$ & $\mathrm{~S}_{3} \mathrm{~S}_{4}$ \\
\hline Total & $\mathbf{6 1 4 , 9 0}$ & & \\
Média & $\mathbf{3 4 , 1 6}$ & & \\
Desvio padrão & $\mathbf{7 , 2 3}$ & & \\
\hline & & &
\end{tabular}


Já foram estudas a topografia do cone medular em macacos (CARVALHOBARROS et al., 2003) e golfinhos de água doce (FETTUCCIA; SIMÕES-LOPES, 2004). Em lobo-guará (Chrysocyon brachyurus), o cone medular foi localizado entre as vértebras $L_{3}$ e $L_{6}$, tendo este $6,5 \mathrm{~cm}$ de comprimento (MACHADO et al., 2002). Já a paca (Agouti paca), apresenta o cone medular entre as vértebras $L_{5}$ e $S_{1}$, tendo comprimento de 5,20 a 5,80cm (SCAVONE et al., 2007). A espécie Arctocephalus australis (lobo marinho) possui o cone medular entre as vértebras $T_{5}$ e $T_{7}$, caracterizando uma topografia diferente das de outros mamíferos, tendo $4,40 \mathrm{~cm}$ de extensão (MACHADO et al., 2003).

Machado e Santos (2008) relatam que no tamanduá-mirim a base do cone é em $L_{1}$ e o ápice em $L_{5} / L_{6}$, tendo 6,5 a $7,0 \mathrm{~cm}$ de comprimento. Machado et al. (2009) relata que no ratão do banhado este se inicia na vértebra $L_{4} / L_{5} e$ termina na $L_{3} / L_{4} / L_{5}$, tendo $2 \mathrm{~cm}$ de comprimento. Lima et al. (2010) estudando o veado-catingueiro (Mazama gouazoubira) verificou que o cone medular inicia-se entre $L_{2}$ e $L_{3}$ e termina em $S_{1} / S_{2}$ e possui comprimento $4,6 \mathrm{~cm}$. Para Gregores et al. (2010) no quati (Nasua nasua) a base do cone medular está na vértebra $L_{5}$ e o ápice na vértebra $S_{3}$, com comprimento entre $5,2 \mathrm{~cm}$ e $5,8 \mathrm{~cm}$. Santos et al., (2011) relatam que em ouriços-cacheiros (Coendou prehensilis) o cone medular estende-se da quarta vértebra lombra $\left(L_{4}\right)$ até a segunda sacral $\left(\mathrm{S}_{2}\right)$, possuindo $5,3 \mathrm{~cm}$ de comprimento.

\section{CONCLUSÕES}

O cone medular de javali (Sus scrofa scrofa) estenderam-se da vértebra $\mathrm{L}_{4}$ até a terceira vértebra sacral $\left(\mathrm{S}_{3}\right)$, com comprimento médio de $34,16 \mathrm{~mm} \pm$ $7,23 \mathrm{~mm}$. Assim, recomenda-se o sítio sacrococcígeno para a realização da anestesia epidural em javali. 


\section{REFERÊNCIAS BIBLIOGRÁFICAS}

ALBUQUERQUE, J. F. G. e et al., Topografia vértebro-medular de cutias (Dasyprocta sp.). In: IV Congresso de Anatomia Del Cono Sur, XX Congresso Brasileiro de Anatomia, XXIII Congresso Chileno de Anatomía, XXXIX Congreso Argentino de Anatomía, e Simpósio Sobre Ensino de Anatomia. Int. J. Morphol. v. 21, n. 1, p. 49-92, mar. 2003b.

ALBUQUERQUE, J. F. G. et al., Topografia vértebro medular do mocó (Kerodon rupestris). In: IV Congresso de Anatomia Del Cono Sur, XX Congresso Brasileiro de Anatomia, XXIII Congresso Chileno de Anatomía, XXXIX Congreso Argentino de Anatomía, e Simpósio Sobre Ensino de Anatomia. Int. J. Morphol. v. 21, n. 1, p. 49-92, mar. $2003 a$.

AMORIM JÚNIOR, A. A. e et al. Topografia do cone medular da preguiça (Choloepus hoffmanni PETERS, 1865 e Bradypus variegatus SHINZ, 1825). In: IV Congresso de Anatomia Del Cono Sur, XX Congresso Brasileiro de Anatomia, XXIII Congresso Chileno de Anatomía, XXXIX Congreso Argentino de Anatomía, e Simpósio Sobre Ensino de Anatomia. Int. J. Morphol. v.21, n. 1, p. 49-92, mar. 2003.

BEDNARSKI, R. M. Manual de Anestesia Veterinária. 3. ed. Porto Alegre: Artes Médicas, 432p. 2001.

BENESCH, F. Eine anathesierungs methode am stehenden rind fur das albrechtsensche verfahren der sterilitatsfehandlung. Wein. Tierarztl. Monatschr. p. 130-134, 1926.

BERGONSO, T. H. D. et al., Valores de referência de uréia e creatinina para javalis (Sus scrofa scrofa, Linnaeus, 1758) em cativeiro. Archives of Veterinary Science. v. 15, n. 4, p. 190195, 2010.

BOHN, F. K. Device for positioning small animals during anesthesia. Laboratory animals, Inglaterra, v. 15, n. 3, p. 289, Sept. 1981.

BOSSI, V. et al. Trattato de anatomia veterinria. Milano: Francesco Vallardi, p. 19, 1909.

BRUNi A. C.; ZIMMERL U. Anatomia Degli Animali Domestici. Italia: Casa Editrici Dottor Francesco Vallardi, 1977. 736p. v.2.

BRUNI, A. C.; ZIMMERL, U. Anatomia degli animali domestici. Milano: Francesco Vallardi, p. $431,1947$.

CARVALHO, R. C. Topografia vertebro-medular e anestesia espinhal em Joboti das "Patas Vermelhas" Geochelone carbonária (SPIX, 1824). São Paulo: USP, 2004. 126f. Dissertação (Mestrado em Ciências) Programa de Pós-graduação em Ciências, Faculdade de Medicina Veterinária e Zootecnia, Universidade de São Paulo, 2004.

CARVALHO-BARROS, R. A. et al. , D. C. O. Constituição do plexo lombar do macaco Cebus apella. Brazilian Journal of Veterinary Research and Animal Science, São Paulo, v. 40, n. 5, p. 373- 381, Feb. 2003.

DYCE, K. M.; SACK, W. O.; WENSING, C. J. G. Tratado de Anatomia Veterinária. 3ed. Rio de Janeiro:Editora Guanabara Koogan, p. 813, 2004.

DYCE, K. M.; SACK, W.O.; WEISING, C.J.G. Tratado de Anatomia Veterinaria. 2 ed. Rio de Janeiro: Guanabara Koogan, p. 186,187, 207-209, 1997. 
ECCO, R.; LAZZARI, A. M.; GUEDES, R. M. C. Pneumonia enzoótica em javalis (Sus scrofa). Pesq. Vet. Bras. v. 29, p. 461-468, 2009.

FETTUCCIA, D. C.; SIMÕES-LOPES, P. C. Morfologia da coluna vertebral do boto cinza, Sotalia guianensis (Cetacea, Delphinidae). Biotemas, v.17, n.2, p.125-148, Out. 2004.

GETTY, R. Sisson/Grossman Anatomia dos animais domésticos. 5ed. Rio de Janeiro: Guanabara Koogan, v.1 e 2, 1986.

GREGORES, G. B. et al., Topografia do cone medular do quati. Biotemas, Montes Claros, v.23, n.2, p.35-42, Fev. 2010.

HABEL, R. E. Guide to the Dissection of the Cow. New York: Cornell Cooperative Society, p. $375,1951$.

HALL L. W. Princípios generales de la anestesia. In: Manual de anestesia de los pequeños animales. 3 ed. Zaragoza: Acribia, p. 1-8, 1992.

HANDERSON, R. A. Epidural anaesthesia in small animals. Auburn Veterinarian, Auburn, v.34, n.1, p.22-35, Fev. 1977.

HOPKINS, G. S. The correlation of anatomy and epidural anesthesia in domestic mammals. Cornell Veterinarian, Ithaca, v.25, n.2, p.263-270, Out. 1935.

HRUBY, J.; DEWY, T. Wild boar. Animal Diversity Web. [2002]. Disponível em: http://animaldiversity.ummz.umich.edu/site/accounts/information/sus_scrofa .html>.Acesso em: $11 / 12 / 2004$.

LIMA, E. M. M.; SANTOS, A. L. Q. Comprimento da medula espinhal e topografia do cone medular em Oryctolagus cuniculus. In Encontro de Iniciação Científica, 7; Uberlândia, MG, 1998. Anais... p. 162.

LIMA, F. C. et al., Topographic anatomy of the spinal cord and vertebromedullary relationships in Mazama gouazoubira Fisher, 1814 (Artiodactyla; Cervidae) Acta Scientiarum. Biological Sciences, Maringá, v.32, n.2, p.189-194, Out. 2010.

MACHADO, G. V.; CAL, J. A.; BIRCK, A. J. Topografia do cone medular no ratão-do-banhado (Myocastor coypus Molina, 1782 - Rodentia: Mammalia). Biotemas, Monte Claros, v.22, n.2, p.117-120, Fev. 2009

MACHADO, G. V.; SANTOS, B. S. Topografia do cone medular no tamanduá-mirim (Tamandua tetradactyla Linnaeus, 1758) (Xenarthra: Myrmecophagidae). Archives of Veterinary Science, Curitiba, v.13, n.2, p.172-175, Fev. 2008.

MACHADO, G. V.; LESNAU, G. G.; BIRCK, A. J. Topografia do cone medular no lobo marinho (Arctocephalus australis Zimmermann, 1783). Arquivos de Ciências Veterinárias e Zoologia da Unipar, Umuarana, v.6, n.1, p.11-14, Out. 2003

MACHADO, G. V.; FONSECA, C. C.; NEVES, M. T. D.; PAULA, T. A. R.; BENJAMIN, L. A. Topografia do cone medular no lobo-guará (Chrysocyum brachyurus Illiger, 1815). Revista Brasileira de Ciências Veterinária, Nitoroi, v.9, n. 2, p.107-109, Sept. 2002.

RAO, G. S. et al., Anatomical studies on the spinal cord segments of the impala (Alpycerus melanus). Zentralblatt fur Veterinarmedizin, Reihe C, Anatomia, Histologia, Embryologia, Berlin, v. 22, n.1, p. 273-278, Jan. 1993. 
RAO, G. S. Anatomic studies on ovine spinal cord. Anatomischer Anzeiger, Deerfield Beach, v. 171, n.3, p.261-264, Out. 1990.

SANTIAGO W.; MACHADO, G. V.; VIZIOLI, V. P.; BARRA, P. H. M. Esqueletopia do cone medular em caprinos mestiços. 1990 Belo Horizonte. Anais. Belo Horizonte: IX Congr. Mineiro de Med. Veterinária, 1990, p.107.

SANTOS, A. L. Q. et al. Topografia do cone medular de Ouriço-cacheiro (Coendou prehensilis, Linnaeus, 1758) (Rodentia). PUBVET, Londrina, V. 5, N. 16, Ed. 163, Art. 1105, 2011.

SANTOS, A. L. Q.; LIMA, E. M. M.; SANTANA, M. I. S. Topografia do cone medular em caprinos da raça Saanen. Arquivos de Ciência Veterinária e Zoologia da UNIPAR, Umuarama, v.4, n.1, p.25-29, Nov. 2001.

SANTOS, A. L. Q.; LIMA, E. M. M. de. Topografia do cone medular em ovinos sem raça definida (Ovis aries LINNAEUS, 1758). Ars Veterinaria, v. 16, n. 3, p. 154-157, 2000.

SANTOS, A. L. Q.; LIMA, E. M. M ; SANTANA, M. I. S. Lenght of spinal cord and topography of medullar cone in rabbit (Oryctogalus auriculus). Bioscience Journal, Uberlândia, MG, v.15, n.2, p.45-62, Mar. 1999.

SCAVONE, A. R. F. et al., Topografia do cone medular da paca (Agouti paca, Linnaeus - 1766). Brazilian Journal of Veterinary Research and Animal Science, São Paulo, v.44, n.3, p.53-57, Feb. 2007.

SEIFERLE E. On the topography of the equine and bovine spinal cord. Zeitschrift für Anatomie und Entwicklungsgeschichte, German, v.110, n.3, p.731-784. Jan. 1951.

SHARMA, D. N.; RAO, G. S. Topography of spinal cord segments in buffalo (Bubalus bubalis). The Indian Journal Animal Science, Nova Deli, v.41, n.2, p.161-165, Nov. 1971.

ST. CLAIR, G.W., HARDENBROOK, H.J. Lombar epidural anestesia in cattle. Journal American Veterinary Medical Association, Schaumburg, v. 129, n. 1, p. 405-409, Nov., 1956. 